\title{
Is SARS-CoV- 2 Strong or Our Body Is Weak?
}

\section{Huang Wei Ling *}

Medical Acupuncture and Pain Management Clinic, Franca, São Paulo, Brazil

"Corresponding author: Huang Wei Ling, Infectious Disease Specialist, General Practitioner, Nutrition Doctor, Acupuncturist, Pain Management, Medical Acupuncture and Pain Management Clinic, Franca, São Paulo, Brazil

Received date: 5 July, 2021 ～Accepted date: 26 July, 2021 | Published date: 29 July, 2021

Citation: Huang WL (2021) Is SARS-CoV- 2 Strong or Our Body Is Weak? J Virol Viral Dis 1(1). doi https://doi.org/10.54289/JVVD2100101

Copyright: (C) 2021 Huang WL, et al. This is an open-access article distributed under the terms of the Creative Commons Attribution License, which permits unrestricted use, distribution, and reproduction in any medium, provided the original author and source are credited.

The author chose to talk about this topic because the way the media talks about the number of deaths that is occurring in everyday life, due to COVID-19, gives the impression that we are facing elephants and human beings' mere ants.

To better understand the process of disease formation, especially of infectious diseases, the author will use older techniques of medicine that currently medicine is still unable to explain.

To understand how our defense system constitutes, the author will explain by another process that is not explained by Western Medicine.

The author will follow the commandments of Hippocrates, father of Medicine, who says that we should consider the oldest medicines before the current practice of Medicine [1]. According to the principles of Chinese medicine, human beings are made of energy. For the whole process of survival and for maintenance all functions, we need energy. Just as for example, the cell phone needs energy to work and needs to be charged every day, and the car to walk, needs to have the intact battery, otherwise do not move either [2].

The human being normally has its natural protection against the onset of diseases or against the invasion of germs that comes from outside $[3,4]$.

All diseases that are caused by external pathogen invasion is called external pathogenic factors invasion by traditional Chinese medicine and SARS-CoV-2 is also considered external pathogenic factor or Xie-Qi $[3,4]$.
For the human being to be healthy, all the energies that bring protection against the invasion of external pathogenic factors must be intact [3].

Food components must be adequate amount, the way of preparation, the proper time to eat each meal and the energy that absorbs all these nutrients must also be adequate also [3]. Furthermore, the breathing process is the second way of capturing energy by the body, bringing oxygen to all cells and systems, and this process should also be intact to maintain health [3].

Contrary to what Western medicine advocates, in traditional Chinese medicine, all internal organs are interconnected and each external sensory organ is commanded by an internal organ [2].

For example, vision is commanded by the Liver (first chakra), hearing is commanded by the Kidney (second chakra), smell is commanded by the Lung (fourth chakra) and communication is commanded by the Heart (third chakra), and taste is commanded by the Spleen (fifth chakra) $[\mathbf{2}, \mathbf{5}]$.

The Kidney energy (second chakra) gives protection against the invasion of the external pathogenic factors (Zheng-Qi) according to traditional Chinese medicine (second chakra) [6, 7].

This organ can have its protective capacity lowered, normally from the age of 30 , when naturally there is a drop in this energy [8].

Kidney energy is also responsible for maintaining the bones, teeth, hearing, reproduction and sexuality [8]. 
In China, doctors to assess energy that exists in the internal organs, they usually measure the pulses in the right and left wrist [5].

In these patients, it can be difficult to measure and it can be very variable the evaluation between doctors. So, the author is using another kind of measurements of the internal energy organs nowadays measuring energy of the chakras' energy centers and in this way, she could measure the energy of the five massive organs (Liver, Heart, Spleen, Lung and Kidney) that are responsible for the generation of energy (Yin, Yang, $Q i$ and Blood) for the maintenance of the human beings' life $[2,9,10]$.

Yin and Yang are two energies produced in the Kidney (second chakra) [8].

Blood is produced in the Spleen (fifth chakra) and Blood flow within the blood vessel is controlled and regulated by the Heart (third chakra) [2].

The $Q i$ or vital energy or prana in Ayurvedic medicine is commanded by the energy of the Liver (first chakra) and by the energy of the Lung (fourth chakra) [11].

In the study carried out by the author evaluating a thousand patients' chakras' energy centers during the period from 2015 to 2020 , she demonstrated that more than $90 \%$ of the patients she attends nowadays do not have energy in the five massive organs (Liver, Heart, Spleen, Lung and Kidney). Therefore, the energy that maintains life is seriously compromised and our body's protective energy, against invasion of external pathogenic factor is absent (in $97 \%$ of the patients evaluated in that period), which is Kidney (second chakra) $[3,4,7]$.

The factors that are entering the body and causing illness are called Xie-Qi [3].

In the article written by Huang (2021) entitled Are We Vaccinating Immunocompetent or Immunocompromised People for COVID? the author describes that when Zheng-Qi is normal and Xie-Qi is low, the patient will be healthy. But when Zheng-Qi is low and Xie-Qi is bigger (proportionally), the patient becomes ill [3].

Therefore, when Zheng-Qi is weak, the Xie-Qi, that normally is not so aggressive, can become a potentially disease-causing person, but not because it is strong but because the energy that commands the disease protection function is weak to protect against the person invasion of external pathogens invasion. And this situation is the form that we are going through these days [3, 7].

SARS-CoV- 2 is not so aggressive but what is damaged are the factors that protects against the invasion of these external pathogens that it is weak (Zheng-Qi) $[3,7]$.

In an article written by Huang (2021) entitled Are We Vaccinating Immunocompetent or Immunocompromised People for COVID? the author proves that in the study she carried out in Brazil, 97\% of the patients she analyzed between 2015 and 2020 were with low Zheng-Qi, but not only the Kidney energy but the entire energy of the body, leading to a state of immunodeficiency and not immunocompetent state [4].

This is caused by the electromagnetic waves that the whole world is exposed to, as in the article written by Huang (2021) entitled Energy Alterations and Chakras Energy Deficiencies and Propensity to SARS-CoV-2 Infection. In this article, the author wrote that there is a pollution between the sky and the earth, generating this global immunosuppression [3].

The other factors that are contributing to keeping low defense energy is the type of medication used to treat pathologies today as the author demonstrated in the article Huang (2021) wrote entitled Why Medications Used Now adays are Harmful to Our Health? [12].

Drugs considered scientific (highly concentrated medications) are causing a change in the vital energy of the human being that is already weak, lowering further and leading to various infectious and non-infectious complications and can lead to death, according to Arndt Shultz Law as showed in the article Huang (2021) wrote entitled Are The Medications That We Are Prescribing To Our Patients Harming Them? [13].

The medications of choice for the treatment of any pathology nowadays are highly diluted medications so that there is no further reduction in vital energy demonstrated in many articles wrote by Huang (2019) such as in the article Why Are Diabetic Patients Still Having Hyperglycemia despite Diet Regulation, Antiglycemic Medication and Insulin? and other article Huang wrote (2020) entitled The Importance of Treating Energy Imbalances and Chakras Replenishment for 
Prevention and Treatment of Cancer, among others [3,9].

Adequate nutrition must be given according to the type of energy imbalance of each patient and the same orientation should not be given to different patients, since each patient is a unique being and must be evaluated individually to receive the orientations and nutrients necessary to rebalance their energy imbalance. The evolution of the outcome in SARSCOV-2 infection can change their course, depending on the type of diet that the patients are receiving, as showed in the article wrote by Huang (2021) entitled Chakras' Energies Deficiencies as the Cause of Dyspnea Post COVID-19 Treatment. In this article, the author said that the patient in the case report one, had dyspnea after 30 days of COVID-19 treatment, using antibiotics and Hydroxychloroquine and was diagnosed, after this treatment, that she had all chakras' energy centers complete lack of energy. One day, after eating pear and tangerine, her dyspnea worsened (due to more energy deficiency caused by the ingestion of these kinds of fruits, leading to more Kidney Yang deficiency) [14].

So, we must give up getting into our minds that the virus is powerful and big and wants to kill us. The author can fell in all prescriptions she is seeing nowadays, to give so much medication to kill the virus. The more we are afraid of him, the more our protective energy will weaken (Zheng-Qi) and the more likely we are for his invasion as showed by Huang (2021) in the article Chakras' Energies Deficiencies as the Cause of Dyspnea Post COVID-19 Treatment. In this article, the author is showing that each massive organ (Kidney, Liver, Heart, Spleen and Lung) have an emotion that they can generate if they are imbalance and each emotion can be imbalanced when there is one specific emotion. In the case of fear, the internal massive organ that they can imbalance is the Kidney (that is the organ responsible for the formation of Zheng-Qi) [14].

To improve our Zheng-Qi, we must drink a lot of water and it cannot be Cold, because Cold water facilitates the invasion of the external pathogenic factor, and could worsen the COVID19 evolution. Sleep a lot and go to bed early preferably at 9 PM, avoid drinking too much coffee, caw's milk, matte tea and soda (because all these drinks can worsen the production of Yin and Yang energy by the Kidney and also, reduce the production of Zheng-Qi). The author (2020) wrote two studies entitled Can We Treat Children with Acute and Chronic Respiratory Tract Infections without Using Antibiotics? and in the article written by Huang (2020) entitled Invasion of Wind and Cold as Cause of Respiratory Tract Infection Outbreak in a School Kinder Garden Group of Kids, the author is demonstrating in these two articles that the cause of all these upper respiratory tract infections were the entrance of the external pathogenic factors (wind and cold) inducing the infectious symptoms $[6,7]$.

The physician should orientate their patients to reduce the sexual practice because having lots of sex, as intense and constant sexual practice, can damage Kidney energy, as showed in the article written by Huang (2020) entitled "Why patients with knee pain still have symptoms despite the use of anti-inflammatory medications" [8].

In the article written by Huang (2021) entitled Energy Alterations and Chakras' Energy Deficiencies and Propensity to SARS-CoV-2 Infection, she is saying that the use of highly concentrated medications should be given with many caution to these patients because can induce more reduction in the vital energy, leading to several complications such as dyspnea, showed in the article written by Huang (2021) entitled Are Pulmonary Manifestations in COVID-19 Really Caused by the Virus? In patients with COVID-19, the use of highly diluted medications is very recommended nowadays and it is the medication of choice to treat COVID19, as the author presented in the International Forum Promoting Homeopathy in March 17 $7^{\text {th }}, 2021$, the study entitled Why Homeopathy in the Prevention and Treatment of SARS-Cov-2 Infection? and also in many other webinars such as in the Webinar on Traditional \& Alternative Medicine, that was held on April 26, 2021. In these presentations, the author state that the use of these kinds of medications can improve the vital energy, and that is why, these kinds of medications are the choice in the treatment of all kinds of diseases nowadays [15].

The use of homeopathies according to the theory Constitutional Homeopathy of the Five Elements based on Traditional Chinese Medicine is the major importance to treat all the symptoms related to COVID-19, with exception of the 
first phase that we need to spell first the entrance of the external pathogenic factors, before using these medications. The medications to use in the first phase of COVID-19 infections are the ones recommended by Hahnemann theory (simillimum) and physician can use COVID-19 nosoed, where homeopathy medications made by COVID-19 patients secretions, diluted in many potencies, to use in therapeutic and also, in prophylaxis of this infection $[16,17]$.

The use of Constitutional Homeopathy of the Five Elements based on Traditional Chinese Medicine, is based on traditional Chinese medicine reasoning and the use of these homeopathies will tone the energy of the 5 massive organs, that are responsible for the production of Yin, Yang, Qi and Blood, that are seriously compromised in almost all the patients nowadays, including patients that has SARS-CoV-2 infection [17].

In this way, we can maintain a higher energy of protection and the consequences of this energy improvement will be reduced rate of viruses entering in our body and causing less harm, because our own defense system will already be fighting against the virus and without the need to take medicine to kill it. This kind of treatment the author is demonstrating in the treatment of variety of infectious problems that are resistance by the use of antibiotics such as in the article Huang (2020) entitled Energies Alterations and Chakras Energies Deficiencies as the Main Cause of Recurrent Urinary Tract Infection Resistant to Antibiotics Treatments. In this article, the author is showing that the adherence of bacteria was caused by the retention of Heat inside the bladder and when we treat this Heat, taking out with apex ear bloodletting and Chinese dietary counseling, the Heat will not be there anymore and the bacteria will not have condition to adhere in the bladder cells [18] .

The use of highly diluted medications in the treatment of all these patients are based on replenishing the chakras' energy centers and the treatment with homeopathies to treat these deficiencies will replenish the chakras' energy centers and will not produce more Heat that would not have the energy disturbances to cause the adherence of the SARS-CoV-2 in the human cells. This reasoning was written by Huang (2021) in the editorial article entitled What have behind in all kinds of infections that we need to know? In this article, the author is saying that all kinds of infection have in common energy deficiencies and Heat retention and COVID-19 patients has these energies disharmonies, as showed in the article entitled Energy Alterations and Chakras' Energy Deficiencies and Propensity to SARS-CoV-2 Infection $[3,18,19]$.

If we use this way of reasoning to control this pandemic, we will be able to return to a more normal life, work normally and the economy will normalize as well, due to the immune system of the population will improve, increasing in this case the Zheng-Qi and the Xie-Qi will be controlled and the Zheng$Q i$ of the human being will fight against this infection, without needing to take medication to "kill the virus" [3].

Only the use of the vaccine as it is currently being done, it may not be as effective as vaccinating immunodepressed people does not generate an adequate vaccine response, since it will not generate the formation of antibodies properly and perhaps, this can explain that the pandemic is not controlled yet if we take only this type of measure alone, as explained by Huang (2021) in the article Are the Vaccines the Only Solution to Control COVID-19 Pandemic? [20].

The problem is deeper than we imagine but if we do not work on the cause and just cover the sun with the sieve, we still will not be solving our problem but we will be facing a problem like Israel today in which in less than a month of returning to life, they are beginning to see returning to admit patients with SARS-CoV-2 infection in the hospital waiting for the fourth wave of COVID-19. The same is occurring in Australia, that after returning to normal life weeks ago, they are facing cases of COVID-19 caused by other strains. So, measures to increase our energy is fundamental now to increase our immune system that is low and using this reasoning, we could fight against the many other variants that SARS-CoV-2 have until today and will have in the future $[21,22]$.

\section{References}

1. Craik EM (2014) The "Hippocratic" Corpus: Content and Context. Routledge (2014): 344.

2. Ling HW (2019) Why Are Diabetic Patients Still Having Hyperglycemia Despite Diet Regulation, Antiglycemic Medication and Insulin? Int J Diabetes Metab Disord 4(2): 114. 
3. Ling HW (2021) Energy Alterations and Chakras' Energy Deficiencies and Propensity to SARS-CoV-2 Infection. Acta Scientific Microbiology 4(4): 167-196.

4. Huang WL (2021) Are We Vaccinating Immunocompetent or Immunocompromised People for COVID 19? J Vaccines Res Vaccin 7: 018.

5. Ling HW (2020) Energies Alterations and Chakras Energies Deficiencies as Underlying Cause of Essential Tremors. Clin Res Neurol 3(2): 1-9.

6. Ling HW (2020) Can We Treat Children With Chronic Respiratory Tract Infections Without Using Antibiotics? Pediatr Res Child Health. 6(2).

7. Ling HW (2020) Invasion of Wind and Cold as Cause of Respiratory Tract Infection Outbreak in a School Kinder Garden Group of Kids. Pediatr Res Child Health. 3(2).

8. Ling HW (2020) Why Patients with Knee Pain Still Have Symptoms Despite the Use of Anti-inflammatory Medications. Int J Orthop Res. 2(2): 21-27.

9. Ling HW (2020) The Importance of Treating Energy Imbalances and Chakras Replenishment for Prevention and Treatment Chakras Replenishment for Prevention and Treatment of Cancer. Adv Cancer Res Clin Imaging 3(1).

10. Ling HW (2021) Chakras' Energies Deficiencies as the Main Cause of Myocardial Infarction without Arterial Obstruction. On J Cardio Res \& Rep 4(5).

11. Ling HW (2018) How Do You Treat Back Pain in Your Practice? Part 2. Med Acupunct. 30(1): 46-53.

12. Ling HW (2021) Why Medications Used Nowadays are Harmful to Our Health? Arch Anesthesiol 4(1).
13. Ling HW (2021) Are the Medications That We Are Prescribing to Our Patients Harming Them? 6(1):11-13.

14. Ling HW (2021) Chakras' Energies Deficiencies as the Cause of Dyspnea Post COVID-19 Treatment. J Infect Dis Case Rep 2(1):1-9.

15. Webinar on Traditional \& Alternative Medicine. April 26, 2021.

16. Ling HW (2021) Chakras' Energy Deficiencies as the Cause of Fatigue Post SARS-CoV-2 Infection Patients Treatment. Acta Sci Microbiol 4(6): 91-108.

17. Ling HW (2020) Constitutional Homeopathy of Five Elements Based on Traditional Chinese Medicine. Acta Sci Med Sci. 4(7): 57-69.

18. Ling HW (2020) Energies Alterations and Chakras Energies Deficiencies as the Main Cause of Recurrent Urinary Tract Infection Resistant to Antibiotics Treatments. Clinic Res Urol 4(3):1-8.

19. Ling HW. What have behind in all kinds of infections that we need to know? Journal of Investigative Oncology 2021; 1(1): 18-21.

20. Ling HW (2021) Are the Vaccines the Only Solution to Control COVID-19 Pandemic?. Vaccines Vacccin 6(1): 000152 .

21. AAFP (2021) Coronavirus Disease 2019 (COVID-19) Daily Research Briefs.

22. CNBC (2021) Australia to halve overseas arrivals as delta variant strains hotel quarantine system. 